\section{P35 The Relationships Between Parental Food Parenting Practices and Child Eating Behavior: A Comparison Between Mothers and Fathers}

Rachel L. Vollmer, PhD, RD, rvollmer@fsmail.bradley.edu, Bradley University, 1501 W. Bradley Ave, Peoria, IL, US 61625

Background: Food parenting practices are recognized as important determinants for child eating behavior. Fathers are underrepresented in studies regarding food parenting and child health; thus, studies are needed that include both mothers and fathers.

Objective: Compare the relationships between food parenting practices and child eating behavior among mothers and fathers of young children.

Study Design, Setting, Participants: This cross-sectional study recruited mothers $(n=127)$ and fathers $(n=118)$ of children $(4.2 \pm 1.3$ years old $)$ to complete surveys (face-to-face and online).

Measurable Outcome/Analysis: Each parent completed the Comprehensive Food Parenting Questionnaire, Children's Eating Behavior Questionnaire, and demographic questions. Linear regression was used to compare the relationships between parental food parenting practices (independent variable) and children's eating behaviors (dependent variable). Parent gender was used as moderator, and child age and gender served as control variables in each regression.

Results: Parent gender was a significant moderator in several relationships between parent food parenting practices and child eating behavior. In the relationship between parental restriction for health $(B=-.14, P=.014)$ and monitoring $(ß=-.13, P=.028)$ and child slowness in eating, the slope of the interaction was significantly higher for mothers, meaning that when mothers and fathers use the same level of restriction for health and monitoring, child slowness in eating is higher for children of mothers. When mothers and fathers used the same level of restriction for weight, child food responsiveness $(B=.13, P=.003)$ and emotional overeating ( $\beta=.12, P=.046)$ was significantly higher for children of fathers

Conclusions: There may be differences in how mothers and fathers implement food parenting practices and/or differences in how these practices impact children. Specifically, for fathers, it seems that the use of restriction for weight is more detrimental for children's eating behaviors compared to when mothers use the same level of restriction for weight.

Funding: Illinois State University College of Applied Science \& Technology.

\section{P36 Hispanic Mothers' Barriers to Making Healthy Eating Changes Within the Family}

AmberHammons,PhD, ahammons@csufresno.edu, California State University, Fresno, 5241 N Maple Ave, Fresno, CA 93740; Fresno Elizabeth Villegas, PhD, NORC at the University of Chicago; Stephanie Sloane, PhD, M.ED, University of Illinois at Urbana-Champaign; Maribel Barragan, RD, University of Illinois at Urbana-Champaign;
Margarita Teran-Garcia, PhD, MD, FTOS, University of Illinois Urbana-Champaign; Norma Olvera, PhD, University of Houston; Kimberly Greder, PhD, Iowa State University; Barbara Fiese, PhD, University of Illinois, Urbana-Champaign

Background: Hispanic adults have the highest rates of overweight and obesity of any racial or ethnic group. Several barriers to healthy eating within the family (eg, lack of time, energy, accessibility, and cost) are well documented. However, few studies have assessed Hispanic mothers' perceived barriers, specifically on implementing healthy eating changes in the family's meals and anticipated family members' responses to healthy eating changes.

Objective: The primary objective of this study was to assess Hispanic mothers' perceived barriers in creating healthy eating changes within the family.

Study Design, Setting, Participants: Sixty-one Mexican-American and Puerto Rican mothers enrolled across 4 states (California, Illinois, Iowa, and Texas) who participated in 13 focus groups. These 4 states were selected due to their locations and concentrations of Mexican-American and Puerto Rican families. Eligibility criteria included being Mexican American or Puerto Rican descent, and having a child between the ages of 6-18.

Measurable Outcome/Analysis: Focus groups were transcribed verbatim and then translated to English by 2 independent research assistants for accuracy purposes. The analysis was guided by grounded theory, using a systematic approach to help patterns in the data to be discovered and identified. Themes were created within and across codes by multiple researchers and representative quotes were selected.

Results: Participating mothers felt that the largest barriers to implementing healthy eating changes within the home would be child and spouse resistance. Mothers largely felt that family members would not welcome healthy changes. The resistance mothers encounter may magnify their workload as several mothers talked about having to make multiple meals in a single mealtime to ensure that everyone was fed.

Conclusions: The present study adds to the literature because the focus is on Mexican, Mexican-American, and Puerto Rican families. Family interventions targeting Hispanic families should include an emphasis on empowering parents and providing strategies to increase the likelihood of all family members eating the same meal.

Funding: USDA.

\section{Nutrition Education Program Design Implementation and Evaluation}

\section{P37 Cook it Fresh - Peer-Led, Plant-Based Cooking Demos for a College Campus Community}

Jeanette Andrade, PhD, LDN, RDN, FAND, jandrade1@ufl. edu, University of Florida, 572 Newell Dr, Gainesville, FL, 32611

Continued on page S33 